\title{
Beamwidth for asymmetric and multilayer semiconductor laser structures
}

\author{
Buus, Jens
}

Published in:

I E E E Journal of Quantum Electronics

Link to article, DOI:

10.1109/JQE.1981.1071157

Publication date:

1981

Document Version

Publisher's PDF, also known as Version of record

Link back to DTU Orbit

Citation (APA):

Buus, J. (1981). Beamwidth for asymmetric and multilayer semiconductor laser structures. I E E E Journal of Quantum Electronics, 17(5), 732-736. https://doi.org/10.1109/JQE.1981.1071157

\section{General rights}

Copyright and moral rights for the publications made accessible in the public portal are retained by the authors and/or other copyright owners and it is a condition of accessing publications that users recognise and abide by the legal requirements associated with these rights.

- Users may download and print one copy of any publication from the public portal for the purpose of private study or research.

- You may not further distribute the material or use it for any profit-making activity or commercial gain

- You may freely distribute the URL identifying the publication in the public portal

If you believe that this document breaches copyright please contact us providing details, and we will remove access to the work immediately and investigate your claim. 


\title{
Beamwidth for Asymmetric and Multilayer Semiconductor Laser Structures
}

\author{
JENS BUUS, MEMBER, IEEE
}

\begin{abstract}
An expression for the far field of the fundamental $\mathrm{TE}_{0}$ mode in an asymmetrical dielectric slab waveguide is derived. By using normalized waveguide parameters, universal plots of the beamwidth are presented. These plots include the obliquity factor correction. Experimental results for symmetrical GaInAsP lasers at wavelengths near 1.3 $\mu \mathrm{m}$ are compared with theoretical predictions by Buus and Adams. Calculated results for the $\mathbf{1 . 5 5} \mu \mathrm{m}$ wavelength are presented. A numerical method for the calculation of the far field for structures where four or more layers must be included is outlined.
\end{abstract}

\section{INTRODUCTION}

$\mathrm{T}$ HE far-field pattern of semiconductor lasers is an important property when the laser is coupled to external structures such as optical fibers. Since the far field is related to the field distribution within the laser, it is also an important experimental tool for determination of the refractive indexes of the layers in the laser structure.

Several approximate formulas for the far-field beamwidth exist for symmetrical structures [1], [2]. In [1] the obliquity factor is included; this is not the case in [2], which gives simple formulas for the case of thin active layers. Instead of attempting to derive simple formulas, we introduce normalized parameters and plot universal curves.

For technological reasons asymmetrical structures may be important for lasers with GaInAsP active regions emitting at wavelengths longer than $1.5 \mu \mathrm{m}$. The beamwidth has been calculated for such structures using a theoretical model for the calculation of the refractive indexes.

More complicated structures (four or more layers) require many normalized parameters and it becomes more convenient to calculate the far field numerically in each case.

The discussion is restricted to the fundamental mode perpendicular to the active layer $\left(\mathrm{TE}_{0}\right)$.

\section{Field Distribution}

The asymmetrical structure considered is shown in Fig. 1.

We introduce a normalized frequency $v$ and an asymmetry factor $a$ [3] (the definition of $v$ differs by a factor of 0.5 from [3]):

$$
\begin{aligned}
& v=k t \sqrt{\Delta \epsilon}, \quad k=\frac{2 \pi}{\lambda}, \quad \Delta \epsilon=n_{1}^{2}-n_{2}^{2}, \\
& a=\frac{n_{2}^{2}-n_{3}^{2}}{\Delta \epsilon} .
\end{aligned}
$$

Manuscript received September 9, 1980, revised December 11, 1980. The author is with the Electromagnetics Institute, Technical University of Denmark, Lyngby, Denmark.
Assuming a propagation factor $e^{-j \beta z}$ and defining a normalized propagation constant $b$ :

$$
b=\frac{n_{\text {eff }}^{2}-n_{2}^{2}}{\Delta \epsilon}, \quad n_{\text {eff }}=\frac{\beta}{k} .
$$

The field distribution can be written

$$
E(x)=\left\{\begin{array}{lr}
\exp \left(\frac{v}{t} \sqrt{a+b}(t+x)\right) & x \leqslant-t \\
A_{1} \cos \left(\frac{v}{t} \sqrt{1-b} x\right)+B_{1} \sin \left(\frac{v}{t} \sqrt{1-b} x\right) \\
A_{2} \exp \left(\frac{v}{t} \sqrt{b}(x-t)\right) & |x|<t \\
& x \geqslant t .
\end{array}\right.
$$

Matching the field and its derivative at $x= \pm t$ determines the coefficients $A_{1}, B_{1}$, and $A_{2}$ in (4) and gives the characteristic equation for the fundamental mode

$$
2 v \sqrt{1-b}=\arctan \sqrt{\frac{b}{1-b}}+\arctan \sqrt{\frac{b+a}{1-b}} .
$$

The far field is mainly determined by the Fourier transform

$$
E^{\prime}(\theta)=\frac{\exp (j k R)}{R} \int_{-\infty}^{\infty} E(x) \exp (-j k x \sin \theta) d x .
$$

This expression was evaluated in [4] using a less convenient notation. Introducing (4) in (6) and using the appropriate expressions for $A_{1}, B_{1}$, and $A_{2}$ gives

$$
\begin{aligned}
\frac{\left|E^{\prime}(\theta)\right|^{2}}{\left|E^{\prime}(0)\right|^{2}}= & \frac{\Delta \epsilon^{2}(1-b)^{2} b(a+b)}{(\sqrt{b(1+a)}+\sqrt{a+b})^{2}} \frac{A^{2}+B^{2}}{C^{2} D F} \\
A= & \sqrt{\Delta \epsilon}(\sqrt{b(1+a)}+\sqrt{a+b)} \cos \alpha \\
& -\sin \theta(\sqrt{1+a}+1) \sin \alpha \\
B= & \sin \theta(\sqrt{1+a}-1) \cos \alpha \\
& -\sqrt{\Delta \epsilon}(\sqrt{a+b}-\sqrt{b(1+a))} \sin \alpha \\
C= & \Delta \epsilon(1-b)-\sin ^{2} \theta \\
D= & \sin ^{2} \theta+b \Delta \epsilon \\
F= & \sin ^{2} \theta+(a+b) \Delta \epsilon \\
\alpha= & k t \sin \theta .
\end{aligned}
$$

For $a=0,(7)$ reduces to the relatively simple expression for 


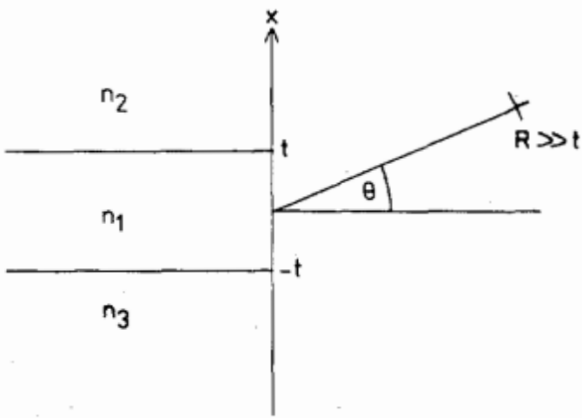

Fig. 1. Asymmetrical slab structure with refractive indexes $n_{1}>n_{2} \geqslant$ $n_{3}$ and thickness $d=2 t$.

the symmetrical slab, see, e.g., [5]-[6]. It is to be noted that (7) is symmetrical in $\theta$; this is the case for all structures where $\Delta \epsilon, a$, and $b$ are real. The imaginary contribution to $\Delta \epsilon$ due to the gain in the active layer is several orders of magnitude smaller than the real part and has no influence on the results.

Numerical trouble in the evaluation of (7) can be encountered at the angle $\theta_{0}$ given by

$$
\theta_{0}=\arcsin \sqrt{\Delta \epsilon(1-b)} \text {. }
$$

Note that $\sin \theta_{0}=n_{1} \sin \theta_{1}, \theta_{1}$ being the propagation direction when the laser structure is treated by a ray description.

From the characteristic (5) it can be shown that $A\left(\theta_{0}\right)=$ $\dot{B}\left(\theta_{0}\right)=0$. Use of L'Hopital's rule then gives

$$
\begin{aligned}
& \left.\frac{A^{2}+B^{2}}{C^{2}}\right|_{\theta=\theta_{0}}=\frac{A^{\prime 2}+B^{\prime 2}}{C^{\prime 2}} \\
& A^{\prime}=\left.\frac{d A}{d \theta}\right|_{\theta=\theta_{0}}=-k t \sqrt{\Delta \epsilon}(\sqrt{b(1+a)}+\sqrt{a+b)} \sin \alpha \cos \theta \\
& \quad-\cos \theta(\sqrt{1+a}+1)(\sin \alpha+\alpha \cos \alpha) .
\end{aligned}
$$

The expressions for $B^{\prime}$ and $C^{\prime}$ are found in a similar way.

In the evaluation of (7) the results from (9) are used in an interval around $\theta_{0}$.

\section{CORRECTION FACTOR}

It has been noted by several authors that the far field is not given by (6), but can be expressed as the product of the Fourier transform and a correction factor [5], [7]-[12]. In [7] this factor was given for a symmetrical structure $\left(n_{3}=n_{2}\right)$ :

$$
g(\theta)=\frac{2 \cos \theta}{\left(\cos \theta+\sqrt{\left.n_{2}^{2}-\sin ^{2} \theta\right)}\right.}\left(n_{\mathrm{eff}}+\sqrt{n_{2}^{2}-\sin ^{2} \theta}\right) .
$$

Later it was shown by Lewin [8], [9] that $n_{\text {eff }}$ should be replaced by an expression with a value close to 1 . Several other expressions have been suggested:

$$
g(\theta)= \begin{cases}\sqrt{\cos \theta} & (\text { see [5]) } \\ \frac{\cos \theta}{n_{\mathrm{eff}}+\cos \theta} & (\text { see [10]) } \\ 1+\cos \theta & (\text { see [11]) } \\ \cos \theta & \text { (see [12]) }\end{cases}
$$

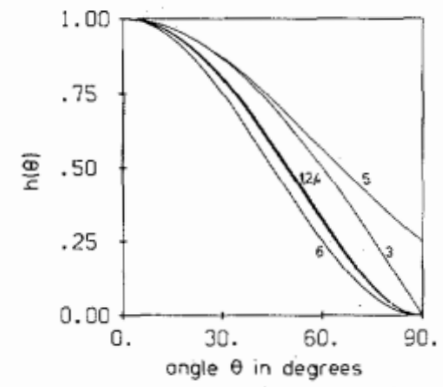

Fig. 2. Normalized correction factor $h(\theta)$ calcualted with $n_{2}=3.4$ and $n_{\text {eff }}=3.5$. 1) (10), 2) (10) with $\left.\left.n_{\text {eff }}=1,3\right)-6\right)(11 \mathrm{a})-(11 \mathrm{~d})$.

The normalized intensity distribution of the far field is then given by

$$
I(\theta)=h(\theta) \frac{\left|E^{\prime}(\theta)\right|^{2}}{\left|E^{\prime}(0)\right|^{2}}
$$

with the normalized correction factor (see Fig. 2)

$$
h(\theta)=\frac{g^{2}(\theta)}{g^{2}(0)} \text {. }
$$

It is seen from Fig. 2 that the simple formula (11b) is a good approximation. The value of $n_{\text {eff }}$ in this formula can be kept constant since the exact value has little influence. This means that the function $h(\theta)$ can be considered as independent of the specific laser structure. The analytical expressions for the beamwidth presented in [1] included a correction factor given by (11d).

\section{RESULTS}

A convenient measure of the far field is the full angular width at half intensity $\theta_{\perp}$. This width is found from (12) by solving

$$
I\left(\frac{\theta_{1}}{2}\right)=\frac{1}{2} \text {. }
$$

Using (11b), with a suitable constant for $n_{\mathrm{eff}}$, in (13), (7), and (12), it is seen that the only parameters needed for the calculation of $\theta_{\perp}$ are $k t, \Delta \epsilon, a$, and $b$. Since $b$ is a function of the normalized frequency $v=k t \sqrt{\Delta \epsilon}$ from (1) and $a$, we see that $\theta_{\perp}$ can be plotted as a function of $k t$ with $\Delta \epsilon$ and $a$ as parameters. Some examples are shown in Fig. 3.

For asymmetrical structures $(a>0)$ a cutoff is present; the cutoff occurs for $v=(\arctan \sqrt{a}) / 2$ [3]. For large values of $k t$, however, the asymmetry has little influence. It is found from the calculations that various combinations of $\Delta \epsilon$ and $a$ which give the same value of $\theta_{\perp}$ (for a given value of $k t$ not close to cutoff) have almost identical far fields, indicating that, in this case, it is sufficient to specify $\theta_{\perp}$ in order to characterize a far field for a given value of $k t$. This also means that it is difficult to estimate $\Delta \epsilon$ and $a$ independently if $\theta_{1}$ is measured for few values of the thickness of the active layer. A possible way to overcome this problem is to find $\theta_{1}$ for small values of the thickness where the influence of the asymmetry is more significant.

If the thickness of layers 2 and 3 in Fig. 1 is much larger than the field penetration depth, the curves of Fig. 3 apply to multilayer structures (e.g., LOC and structures with thick antimeltback layers). 


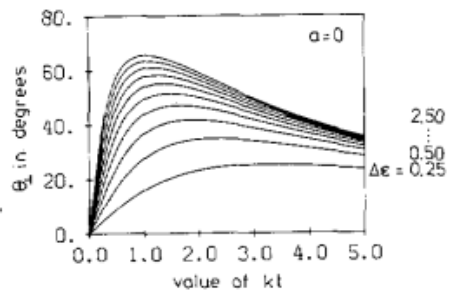

(a)

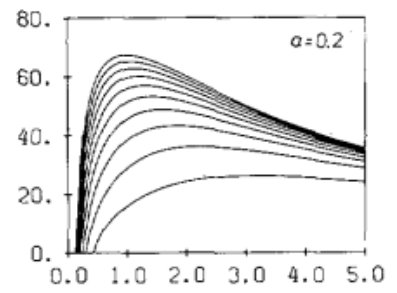

(b)

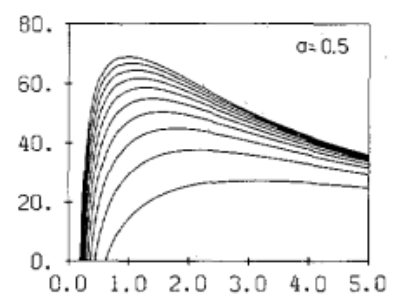

(c)

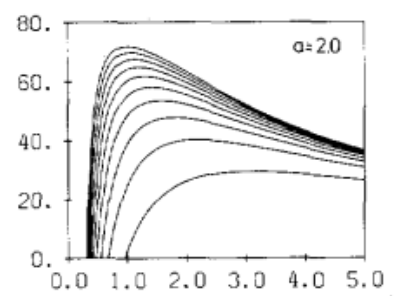

(d)

Fig. 3. Beamwidth $\theta_{\perp}$ in degrees as function of $k t$ with the permittivity difference $\Delta \epsilon$ and asymmetry factor $a$ as parameters. The correction factor is included.

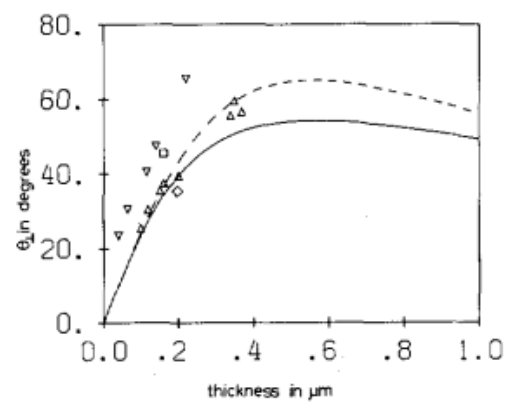

Fig. 4. Beamwidth as function of the thickness of the active layer in $\mu \mathrm{m}$ for symmetrical GalnAsP lasers emitting near $1.3 \mu \mathrm{m}$. The full curve includes the correction factor, the broken curve is calculated without correction. The refractive index model from [13] is used. Experimental points: $\Delta \lambda=1.3 \mu \mathrm{m}[15], \nabla \lambda=1.3 \mu \mathrm{m}[16], \square \lambda=$ $1.26 \mu \mathrm{m} \mathrm{[17],} \mathrm{and} \diamond \lambda=1.28 \mu \mathrm{m} \mathrm{[18]}$.

\section{Application to GaInAsP LASERS}

Recently a model for the refractive index of GaInAsP compounds was suggested [13]. This model was used in [14]; the interpolation formulas, however, were different, hence different index values were predicted. Measurements of the beamwidth make it possible to test the theoretical predictions. Fig. 4

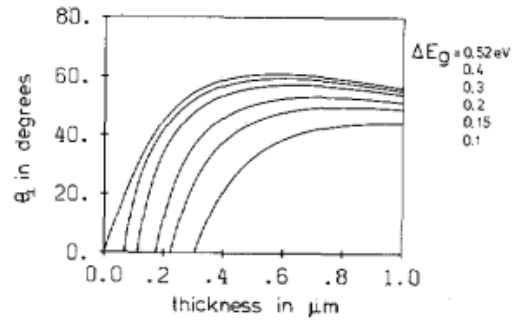

Fig. 5. Beamwidth for asymmetrical GaInAsP lasers emitting at 1.55 $\mu \mathrm{m}$ as function of the thickness of the active layer in $\mu \mathrm{m}$ and with the bandgap difference $\Delta E_{G}$ as parameter. The refraction index model from [13] is used.

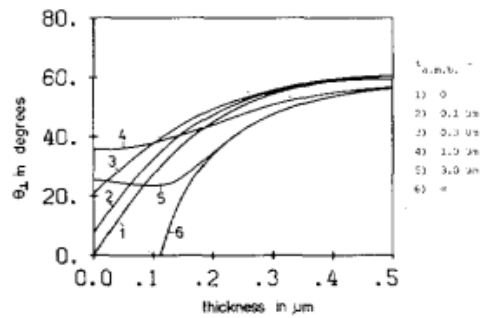

Fig. 6. Beamwidth for GaInAsP lasers, with antimeltback layer, emitting at $1.55 \mu \mathrm{m}$ as function of the thickness of the active layer in $\mu \mathrm{m}$ and with the thickness of the antimeltback layer as parameter. Calculated for $\Delta E_{G}=E_{G_{a m b}}-E_{G_{a c t}}=0.3 \mathrm{eV}$ using the refractive index model from [13].

shows some theoretical (calculated with, as well as without, the correction factor) and experimental results [15]-[18] for symmetrical structures.

Most of the experimental results are obtained for wavelengths near $1.3 \mu \mathrm{m}$ and show large scatter; experiments with DBR lasers at this wavelength [14] give $\Delta \epsilon \approx 2$, whereas [13] gives $\Delta \epsilon \approx 1.4$. For an active layer thickness of $0.2 \mu \mathrm{m}$ these values correspond to the beamwidth $\sim 50^{\circ}$ and $\sim 40^{\circ}$, respectively. It is evident that more experimental results are needed in order to determine the refractive index precisely; also, results for a wavelength longer than $1.3 \mu \mathrm{m}$ will be of interest.

Many of the lasers operating at $1.55 \mu \mathrm{m}$ have consisted of an asymmetrical $\mathrm{Ga}_{z} \mathrm{In}_{1-z} \mathrm{As}_{w} \mathrm{P}_{1-w} / \mathrm{Ga}_{x} \mathrm{In}_{1-x} \mathrm{As}_{y} \mathrm{P}_{1-y} / \mathrm{InP}$ structure in order to avoid meltback of the active layer. The refractive index of the p-layer can be calculated as a function of the bandgap difference $\Delta E_{G}=E_{G_{p}}-E_{G_{\text {act }}}$; this allows the beam width $\theta_{\perp}$ to be calculated as a function of the thickness of the active layer using $\Delta E_{G}$ as a parameter. In this structure both $\Delta \epsilon$ and the asymmetry factor $a$ depend on $\Delta E_{G}$. The results are shown in Fig. 5; experimental results have not been reported yet.

The results shown in Fig. 5 are based on the assumption that the antimeltback layer is thick. If this is not the case, four layers must be included in the calculation and the resulting large number of normalized parameters makes it impractical to plot universal curves. Instead, the far field can be calculated numerically in each case, e.g., using the method described in the appendix. Results for lasers emitting at $1.55 \mu \mathrm{m}$ with $\Delta E_{G}=E_{G_{\text {am b }}}-E_{G_{\text {act }}}=0.3 \mathrm{eV}$ are shown in Fig. 6; the thickness of the antimeltback layer is used as a parameter.

The same numerical method has been applied to the strip buried structure $(\lambda=1.3 \mu \mathrm{m})$ described in [19]. The beam- 


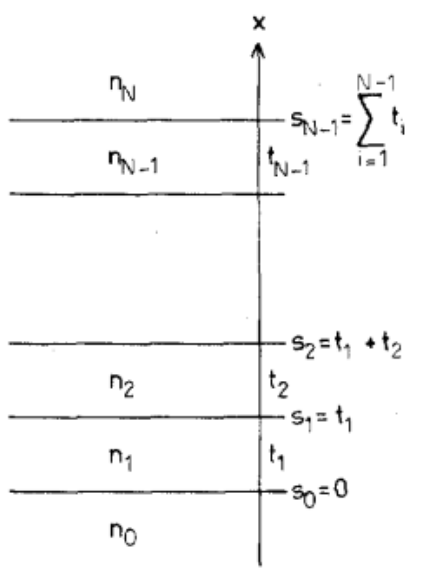

Fig. 7. Geometry of multilayer structure.

width was found to be $33^{\circ}$, in good agreement with the experimental result of $30^{\circ}$. It should be noted that this laser must be treated as a four-layer structure. The fundamental mode in the corresponding three-layer structure with an infinitely thick antimeltback layer is below cutoff.

\section{CONCLUSION}

It is shown that the far field for the fundamental mode perpendicular to the active layer from an asymmetrical laser structure can be found from an analytical expression which only contains a few waveguide parameters. When this expression is combined with a refractive index model it is possible to predict the beamwidth for various lasers. Comparison of the theory and the experiments then serves as a check of the refractive index model.

The measured beamwidths for symmetrical GaInAsP lasers emitting near $1.3 \mu \mathrm{m}$ show large scatter and further results are needed before the refractive index of the active layer can be determined precisely. The calculated results show the importance of the correction factor. Theoretical results for structures with four or more layers can be found using a numerical method.

\section{APPENDIX}

For the multilayer structure shown in Fig. 7 the field is written

$$
\begin{aligned}
E_{i}(x)= & A_{i} \exp \left(\sqrt{\beta^{2}-\left(n_{i} k\right)^{2}}\left(x-s_{i-1}\right)\right) \\
+ & B_{i} \exp \left(-\sqrt{\beta^{2}-\left(n_{i} k\right)^{2}}\left(x-s_{i-1}\right)\right) \\
& s_{i-1}<x \leqslant s_{i} \quad i=0,1, \cdots N .
\end{aligned}
$$

The boundary conditions are

$$
\begin{aligned}
& E_{i}\left(s_{i}\right)=E_{i+1}\left(s_{i}\right) \\
& \left.\frac{d E_{i}(x)}{d x}\right|_{s_{i}}=\left.\frac{d E_{i+1}(x)}{d x}\right|_{s_{i}} \\
& B_{0}=0 \\
& A_{N}=0 . \\
& A_{0} \text { arbitrary. }
\end{aligned}
$$

This set of equations is simple to solve numerically. Starting with $B_{0}=0, A_{0}=1$, and a startguess for $\beta$, the coefficients
$A_{i}$ and $B_{i}$ are found successively using the boundary conditions. This procedure is repeated until a value of $\beta$ giving $A_{N}=0$ is found; this also provides the values of the coefficients $A_{i}, B_{i}$, and the power fraction in each layer can be found.

The Fourier transform is

$$
E^{\prime}(\theta)=\sum_{i=0}^{N} \int_{s_{i-1}}^{s_{i}} E_{i}(x) \exp (-j k \sin \theta x) d x
$$

hence

$$
\begin{aligned}
E^{\prime}(\theta)= & \frac{1}{q_{0} k-j k \sin \theta}+\frac{B_{N}}{q_{N} k+j k \sin \theta} \exp \left(-j k \sin \theta s_{N-1}\right) \\
& +\sum_{i=1}^{N-1}\left\{\frac { A _ { i } } { q _ { i } k - j k \operatorname { s i n } \theta } \left[\exp \left(-j k \sin \theta s_{i}+q_{i} k t_{i}\right)\right.\right. \\
& \left.-\exp \left(-j k \sin \theta s_{i-1}\right)\right] \\
& -\frac{B_{i}}{q_{i} k+j k \sin \theta}\left[\exp \left(-j k \sin \theta s_{i}-q_{i} k t_{i}\right)\right. \\
& \left.\left.-\exp \left(-j k \sin \theta s_{i-1}\right)\right]\right\} \\
& q_{i}=\sqrt{\left(\frac{\beta}{k}\right)^{2}-n_{i}^{2}} .
\end{aligned}
$$

The far field is found using (A4) in (12).

\section{ACKNOWLEDGMENT}

The author wishes to thank L. T. Pedersen of the Electromagnetics Institute for programming work and useful suggestions.

\section{REFERENCES}

[1] D. Botez and M. Ettenberg, "Beamwidth approximations for the fundamental mode in symmetric double-heterojunction lasers," IEEE J. Quantum Electron., vol. QE-14, pp. 827-830, Nov. 1978.

[2] W. P. Dumke, "The angular beam divergence in double-heterojunction lasers with very thin active regions," IEEE J. Quantum Electron., vol. QE-11, pp. 400-402, July 1975.

[3] H. Kogelnik and V. Ramaswamy, "Scaling rules for thin-film optical waveguides," Appl. Opt., vol. 13, pp. 1857-1862, 1974.

[4] G. D. Henshall and J. E. A. Whiteaway, "Far field emission patterns of single heterostructure GaAs lasers," Electron. Lett., vol. 10, pp. 326-327, 1974.

[5] P. A. Kirkby and G. H. B. Thompson, "The effect of double heterojunction waveguide parameters on the far field emission patterns of lasers," Opt. Electron., vol. 4, p. 323-324, 1972.

[6] H. C. Casey, Jr., M. B. Panish, and J. L. Merz, "Beam divergence of the emission from double-heterostructure injection lasers," J. Appl. Phys., vol. 44, pp. 5470-5475, 1973.

[7] G. A. Hockham, "Radiation from a solid-state laser," Electron. Lett., vol. 9, pp. 389-391, 1973.

[8] L. Lewin, "Obliquity factor for radiation from solid-state laser," Electron. Lett., vol. 10, pp. 134-135, 1974.

[9] L. Lewin, "A method for the calculation of the radiation-pattern and mode-conversion properties of a solid-state heterojunction laser," IEEE Trans. Microwave Theory Techn., vol. MTT-23, pp. 576-585, July 1975 .

[10] J. K. Butler and J. Zoroofchi, "Radiation fields of GaAs-(AlGa)As injection lasers," IEEE J. Quantum Electron., vol. QE-10, pp. 809-815, Oct. 1974.

[11] P. J. de Waard, "Calculation of the far-field halfpower width and the mirror reflection coefficients of double-heterostructure lasers," Electron. Lett., vol. 11, pp. 11-12, 1975.

[12] H. C. Casey Jr. and M. B. Panish, Heterostructure Lasers, Part A. New York: Academic, 1978, pp. 71-75.

[13] J. Buus and M. J. Adams, "Phase and group indices for double heterostructure lasers," IEE Solid-State Electron. Dev., vol. 3 , pp. 189-195, 1979. 
[14] K. Utaka, Y. Suematsu, K. Kobayashi, and H. Kawanishi, "GaInAsP/InP integrated twin guide lasers with first order Bragg reflectors at $1.3 \mu \mathrm{m}$ wavelength," Japan. J. Appl. Phys., vol. 19, pp. 137-140, 1980 .

[15] G. D. Henshall and P. D. Greene, "Growth and characteristics of GaInAsP/InP double heterostructure lasers," IEE Solid-State Electron. Dev., vol. 3, pp. 174-178, 1979.

[16] Y. Itaya, Y. Suematsu, S. Katayama, K. Kishino, and S. Arai, "Low threshold current density (100) GaInAsP/InP double heterostructure lasers for wavelength $1.3 \mu \mathrm{m}$," Japan. J. Appl. Phys., vol. 18, pp. 1795-1805, 1979.

[17] G. M. Olsen, C. J. Nuese, and M. Ettenberg, "Low threshold 1.25 $\mu \mathrm{m}$ vapour grown InGaAsP cw lasers," Appl. Phys. Lett., vol. 34, pp. 262-264, 1979.

[18] K. Oe, S. Ando, and K. Sugiyama, "GaInAsP/InP planar strip lasers prepared by using sputtered $\mathrm{SiO}_{2}$ film on a $\mathrm{Zn}$ diffusion mask," J. Appl. Phys., vol. 51, pp. 43-49, 1980.

[19] R. J. Nelson, P. D. Wright, P. A. Barnes, R. L. Brown, T. Cella, and R. G. Sobers, "High-output power InGaAsP $(\lambda=1.3 \mu \mathrm{m})$ strip-buried heterostructure lasers," Appl. Phys. Lett., vol. 36, pp. $358-360,1980$.

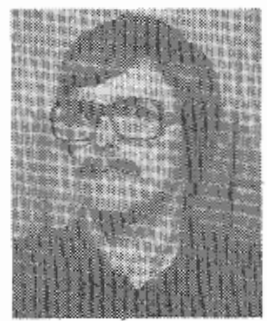

Jens Buus (S'76-M'79) was born in Copenhagen, Denmark, on August 8, 1952. He received the M.Sc. and Ph.D. degrees from the Technical University of Denmark, Lyngby, while doing research on the modeling of the properties of semiconductor lasers.

From March to June of 1979 he was a Research Associate at the University of Wales, Cardiff, Wales. Since August of 1979 he has been a Post Doctoral Research Associate at the Electromagnetics Institute, Technical University of Denmark, working on semiconductor lasers, in particular, waveguiding problems.

\title{
Channeled Substrate Nonplanar Laser Analysis Part I: Formulation and the Plano-Convex Waveguide Laser
}

\author{
WILLIAM STREIFER, FELLOW, IEEE, ROBERT D. BURNHAM, MEMBER, IEEE, \\ AND DON R. SCIFRES, FELLOW, IEEE
}

\begin{abstract}
An analysis of diode lasers with lateral thickness variations in the active and/or guiding layers is presented. The technique, which applies both at and above threshold, models the current flow from the stripe contact, the charge diffusion within the active region, the modal wave propagation, and the cavity condition, self-consistently, such that the phenomena of spatial hole burning in the charge distribution by the modal intensity and the changes in the complex refractive index produced by the charges are accounted for simultaneously. Results are obtained for threshold current as well as laser behavior above threshold, including the charge density distribution, the mode patterns, the differential quantum efficiency, and the lowest order mode power at which the laser begins oscillating in more than one spatial transverse mode. A study of the plano-convex waveguide laser is described in detail and an Appendix discussing the mathematical methods is included.
\end{abstract}

\section{INTRODUCTION}

W ITHIN the past few years interest in diode lasers which incorporate some degree of lateral waveguiding other than a spatially limited extent of injected charge has increased substantially. Various lasers of this type exist [1]-[3] and a particularly noteworthy example is the channeled substrate

Manuscript received September 23, 1980; revised December 15, 1980. The authors are with the Xerox Palo Alto Research Center, Palo Alto, CA 94306.

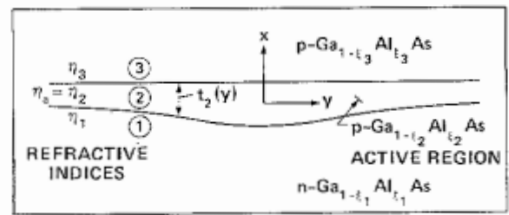

Fig. 1. Schematic of a three-region laser with a laterally tapered active layer.

planar (CSP) device [3]. This laser utilizes substrate radiation at the lateral boundaries of the mode to decrease the effective refractive index there and thus introduce real refractive index lateral waveguiding. Another class of laser achieves the same result by employing lateral thickness variations in the active and/or cladding regions [4]-[10]. In these devices the thickness variations themselves produce a maximum of the real equivalent refractive index at the center $y=0$ and, in contrast to the CSP laser, radiation losses need not be utilized. The three-region version of the device is illustrated in Fig. 1 [9], but both four- [10] and five- [6], [8] layer implementations have been reported and the analysis described herein applies to these devices as well.

As a consequence of the equivalent refractive index maxi- 